To appear in Optimization Methods \& Software

Vol. 00, No. 00, Month 20XX, 1-15

\title{
Dynamic optimization of dual-mode hybrid systems with state-dependent switching conditions
}

\author{
Eunice Blanchard ${ }^{\mathrm{a}}$, Ryan Loxton $^{\mathrm{a} *}$, and Volker Rehbock ${ }^{\mathrm{a}}$ \\ ${ }^{a}$ Department of Mathematics and Statistics, Curtin University, Perth, Australia \\ (Received 00 Month 20XX; final version received 00 Month 20XX)
}

\begin{abstract}
This paper presents a computational approach for optimizing a class of hybrid systems in which the state dynamics switch between two distinct modes. The times at which the mode transitions occur cannot be specified directly, but are instead governed by a state-dependent switching condition. The control variables, which should be chosen optimally by the system designer, consist of a set of continuous-time input signals. By introducing an auxiliary binaryvalued control function to represent the system's current mode, we show that any dualmode hybrid system with state-dependent switching conditions can be transformed into a standard dynamic system subject to path constraints. We then develop a computational algorithm, based on control parameterization, the time-scaling transformation, and an exact penalty method, for determining the optimal piecewise constant input signals for the original hybrid system. A numerical example on cancer chemotherapy is included to demonstrate the effectiveness of the proposed algorithm.
\end{abstract}

Keywords: Dynamic Optimization; Hybrid Systems; State-dependent Switching; Control Parameterization; Time-scaling Transform; Exact Penalty Method

AMS Subject Classification: 49M37; 65K10; 90C30

\section{Introduction}

A hybrid system is a system that involves both time-driven and event-driven dynamics $[2,4,14,17,23]$. The event-driven dynamics typically cause switches in the time-driven dynamics (which are often described by differential equations). Such switches can be classified into two types: controlled switches and autonomous switches. Controlled switches are triggered externally (for example, by changing a gear or switch) and can be manipulated directly by the system operator. Autonomous switches, on the other hand, occur implicitly when the state trajectory crosses from one region in the state space to another; they cannot be controlled directly.

Dynamic optimization problems involving hybrid systems have received significant attention in the research literature. Most papers, especially those discussing computational techniques, focus on hybrid systems with controlled switches [7]. In the present paper, we instead focus on hybrid systems with autonomous switches. Dynamic optimization problems for such systems arise in many practical applications such as medical science $[12,13]$, power engineering [15], chemical process control [4], and robotics [1].

For hybrid systems with controlled switches, most of the available optimization techniques rely on the assumption that the system dynamics are continuously differentiable

\footnotetext{
*Corresponding author. Email: r.loxton@curtin.edu.au
} 
with respect to the system's state (although they are discontinuous with respect to time due to the dynamics changing at the switching time points) [7]. This assumption is clearly violated for hybrid systems with autonomous switchings: the dynamic equations may change discontinuously for small perturbations in the state. Nevertheless, it is common to treat hybrid systems with autonomous switches the same as hybrid systems with controlled switches, disregarding the implicit influence of the control parameters on the autonomous switching mechanism. Consider, for example, the cancer chemotherapy problem in references [11-13]. This problem involves a hybrid system with two modes - one mode is active when the drug concentration is below a given threshold, the other mode is active when the drug concentration is above the threshold. This problem was solved in [11-13] using gradient-based optimization techniques that require the state dynamics to be continuously differentiable functions of the state. Since this assumption is violated for hybrid systems with autonomous switches (of which the dynamics in the cancer problem are an example), the solutions obtained in [11-13] are not guaranteed to be optimal.

A similar gradient-based optimization approach was used in references $[15,19]$ to solve an optimal discrete-valued control problem for hybrid power systems. This approach uses standard formulae for computing the cost and constraint gradients in optimal control (see $[7,8]$ ), but the continuous differentiability assumptions underlying these formulae are violated here because the dynamic system includes an autonomous switching mechanism. In reference [1], proper gradient formulae for hybrid systems with autonomous switching mechanisms are derived. However, these formulae are only applicable to simple hybrid systems in which the control parameters only influence the switching surfaces (not the dynamic equations), there are no continuous-time input signals, and there are no constraints on the system.

In this paper, we consider a more general class of hybrid systems with state-dependent switching conditions. We allow canonical and continuous inequality constraints and we also cater for time-varying input signals. The paper is organized as follows. In Section 2, we define the dynamic optimization problem under consideration, which involves a hybrid system with two modes. In Section 3, we introduce a binary-valued control function to govern the switching between the two modes, and then replace the binary constraints with equivalent constraints defined over a continuous range. In Section 4, we use the well-known control parameterization and time-scaling methods $[3,8,20]$ to derive an approximate finite-dimensional problem. We then apply an exact penalty approach in Section 5 to transform the resulting problem into a form that can be readily solved using the optimal control software MISER 3.3 [5]. Section 6 reports numerical results obtained by applying our new approach to the cancer chemotherapy problem in [11-13].

\section{Problem formulation}

Consider the following hybrid control system, defined on the time horizon $[0, T]$, in which the dynamics depend on the location of the state within the state space:

$$
\dot{\boldsymbol{x}}(t)= \begin{cases}\boldsymbol{f}^{1}(\boldsymbol{x}(t), \boldsymbol{u}(t)), & \text { if } \boldsymbol{x}(t) \in \Omega_{1}, \\ \boldsymbol{f}^{2}(\boldsymbol{x}(t), \boldsymbol{u}(t)), & \text { if } \boldsymbol{x}(t) \in \Omega_{2},\end{cases}
$$

where

- $\boldsymbol{x}(t) \in \mathbb{R}^{n}$ is the state vector at time $t$

- $\boldsymbol{u}(t) \in \mathbb{R}^{r}$ is the control vector at time $t$; 
- $\Omega_{1}$ and $\Omega_{2}$ are given regions with disjoint interiors such that $\Omega_{1} \cup \Omega_{2}=\mathbb{R}^{n}$; and - $f^{1}, f^{2}: \mathbb{R}^{n} \times \mathbb{R}^{r} \rightarrow \mathbb{R}^{n}$ are given continuously differentiable functions.

According to equation (1), the system dynamics switch when the state moves from $\Omega_{1}$ to $\Omega_{2}$, or from $\Omega_{2}$ to $\Omega_{1}$. Thus, unlike in conventional switched systems, the switching times here are not independent decision variables; they are instead generated implicitly by the state trajectory.

We assume that $\Omega_{1}$ and $\Omega_{2}$ can be defined as follows:

$$
\Omega_{1}=\left\{\boldsymbol{x} \in \mathbb{R}^{n}: \omega(\boldsymbol{x}) \geq 0\right\}, \quad \Omega_{2}=\left\{\boldsymbol{x} \in \mathbb{R}^{n}: \omega(\boldsymbol{x}) \leq 0\right\}
$$

where $\omega: \mathbb{R}^{n} \rightarrow \mathbb{R}$ is a given continuously differentiable function. The interiors of these regions are defined by

$$
\operatorname{int}\left(\Omega_{1}\right)=\left\{\boldsymbol{x} \in \mathbb{R}^{n}: \omega(\boldsymbol{x})>0\right\}, \quad \operatorname{int}\left(\Omega_{2}\right)=\left\{\boldsymbol{x} \in \mathbb{R}^{n}: \omega(\boldsymbol{x})<0\right\} .
$$

The initial condition for system (1) is

$$
\boldsymbol{x}(0)=\boldsymbol{x}^{0},
$$

where $\boldsymbol{x}^{0} \in \mathbb{R}^{n}$ is a given initial state.

Let

$$
U=\left\{\left[u_{1}, \ldots, u_{r}\right]^{\top} \in \mathbb{R}^{r}: u_{j}^{\min } \leq u_{j} \leq u_{j}^{\max }, j=1, \ldots, r\right\}
$$

where, for each $j=1, \ldots, r, u_{j}^{\min }$ and $u_{j}^{\max }$ are given constants such that $u_{j}^{\min }<u_{j}^{\max }$. The set $U \subset \mathbb{R}^{r}$ is called the control restraint set. Any piecewise continuous function $\boldsymbol{u}:[0, T] \rightarrow \mathbb{R}^{r}$ such that $\boldsymbol{u}(t) \in U$ for all $t \in[0, T]$ is called an admissible control. Let $\mathcal{U}$ be the class of all such admissible controls.

For a given $\boldsymbol{u} \in \mathcal{U}$, let $\boldsymbol{x}(\cdot \mid \boldsymbol{u})$ be the corresponding state trajectory that satisfies the dynamic equations (1) and the initial condition (2). We assume that, on the boundary between the two regions $\Omega_{1}$ and $\Omega_{2}$,

$$
\boldsymbol{f}^{1}(\boldsymbol{x}(t), \boldsymbol{u}(t))=\boldsymbol{f}^{2}(\boldsymbol{x}(t), \boldsymbol{u}(t)), \quad \boldsymbol{x}(t) \in \Omega_{1} \cap \Omega_{2} .
$$

This ensures that the dynamics are well-defined on the boundary (since the state trajectory may "slide" along the switching surface defined by $\omega(\boldsymbol{x})=0$ for some non-negligible time interval).

We impose two types of constraints on the state trajectory: canonical constraints and continuous inequality constraints. The canonical constraints (of both equality and inequality type) are expressed mathematically as follows:

$$
g_{v}(\boldsymbol{u})=\sum_{k=1}^{m} \Phi_{v, k}\left(\boldsymbol{x}\left(\tau_{k} \mid \boldsymbol{u}\right)\right)+\int_{0}^{T} \mathcal{L}_{v}(\boldsymbol{x}(t \mid \boldsymbol{u}), \boldsymbol{u}(t)) d t \begin{cases}=0, & v=1, \ldots, p_{e} \\ \geq 0, & v=p_{e}+1, \ldots, p,\end{cases}
$$

where $\Phi_{v, k}: \mathbb{R}^{n} \rightarrow \mathbb{R}, v=1, \ldots, p, k=1, \ldots, m$, and $\mathcal{L}_{v}: \mathbb{R}^{n} \times \mathbb{R}^{r} \rightarrow \mathbb{R}, v=1, \ldots, p$, are continuously differentiable functions and $\tau_{1}, \ldots, \tau_{m}$ are given characteristic times satisfying

$$
0=\tau_{0}<\tau_{1}<\tau_{2}<\cdots<\tau_{m} \leq T .
$$


Note that the canonical form (4) can model many constraints of practical interest, including interior-point constraints and terminal state constraints [8].

Continuous inequality constraints are expressed mathematically by

$$
h_{v}(\boldsymbol{x}(t \mid \boldsymbol{u})) \geq 0, \quad t \in[0, T], \quad v=1, \ldots, q,
$$

where $h_{v}: \mathbb{R}^{n} \rightarrow \mathbb{R}, v=1, \ldots, q$, are given continuously differentiable functions. Such constraints impose restrictions on the system at every point in the time horizon.

We define a cost function in the same form as the canonical functions in (4):

$$
g_{0}(\boldsymbol{u})=\sum_{k=1}^{m} \Phi_{0, k}\left(\boldsymbol{x}\left(\tau_{k} \mid \boldsymbol{u}\right)\right)+\int_{0}^{T} \mathcal{L}_{0}(\boldsymbol{x}(t \mid \boldsymbol{u}), \boldsymbol{u}(t)) d t
$$

where $\Phi_{0, k}: \mathbb{R}^{n} \rightarrow \mathbb{R}, k=1, \ldots, m$, and $\mathcal{L}_{0}: \mathbb{R}^{n} \times \mathbb{R}^{r} \rightarrow \mathbb{R}$ are given continuously differentiable functions.

Our optimization problem is defined below.

Problem 1 Choose an admissible control $\boldsymbol{u} \in \mathcal{U}$ to minimize the cost function (6) subject to the hybrid system dynamics (1), the initial condition (2), the canonical constraints (4), and the continuous inequality constraints (5).

\section{Problem transformation}

The system dynamics in (1) are non-smooth because of the state-dependent switching mechanism. Most optimization methods for hybrid systems are only applicable when the switching mechanism is time-dependent and not state-dependent. Therefore, we introduce a new binary-valued decision function $z(t)$ defined as follows:

$$
z(t) \begin{cases}=1, & \text { if } \boldsymbol{x}(t) \in \operatorname{int}\left(\Omega_{1}\right), \\ =0, & \text { if } \boldsymbol{x}(t) \in \operatorname{int}\left(\Omega_{2}\right), \\ \in\{0,1\}, & \text { if } \boldsymbol{x}(t) \in \Omega_{1} \cap \Omega_{2} .\end{cases}
$$

The choice of whether $z(t)=1$ or $z(t)=0$ when $\boldsymbol{x}(t) \in \Omega_{1} \cap \Omega_{2}$ is arbitrary. Since we are assuming that the dynamic equations for $\Omega_{1}$ and $\Omega_{2}$ are identical on the boundary between the two regions (see (3)), we can write (1) as

$$
\dot{\boldsymbol{x}}(t)=z(t) \boldsymbol{f}^{1}(\boldsymbol{x}(t), \boldsymbol{u}(t))+(1-z(t)) \boldsymbol{f}^{2}(\boldsymbol{x}(t), \boldsymbol{u}(t)), \quad t \in[0, T] .
$$

Standard nonlinear optimization algorithms such as interior-point and sequential quadratic programming methods are designed to solve continuous optimization problems. Such methods cannot handle discrete variables in the form of (7). Therefore, to proceed, we drop the binary requirements and consider $z(t)$ to be a continuous-valued function subject to the following bound constraints:

$$
0 \leq z(t) \leq 1, \quad t \in[0, T]
$$

Let $\mathcal{Z}$ denote the set of all piecewise continuous functions defined on $[0, T]$ and satisfying inequality (9). Furthermore, let $\boldsymbol{x}(\cdot \mid \boldsymbol{u}, z)$ denote the solution of (8) and (2) corresponding 
to pair $(\boldsymbol{u}, z) \in \mathcal{U} \times \mathcal{Z}$. To ensure that $z(t)$ is consistent with (7), we impose the following additional constraints:

$$
z(t)(z(t)-1) \geq 0, \quad t \in[0, T]
$$

and

$$
z(t) \omega(\boldsymbol{x}(t \mid \boldsymbol{u}, z))-(1-z(t)) \omega(\boldsymbol{x}(t \mid \boldsymbol{u}, z)) \geq 0, \quad t \in[0, T] .
$$

The following result shows that (9)-(11) are equivalent to (7).

Proposition 3.1 Let $\boldsymbol{u} \in \mathcal{U}$ and $z \in \mathcal{Z}$ be given. Then $(\boldsymbol{u}, z)$ satisfies definition (7) if and only if $(\boldsymbol{u}, z)$ satisfies constraints (9)-(11).

Proof. If $(\boldsymbol{u}, z)$ satisfies (7), then obviously (9)-(11) are also satisfied. Thus, we focus on the reverse implication. Suppose that (9)-(11) hold. Then it follows from (10) that for all $t \in[0, T]$, either $z(t) \geq 1$ or $z(t) \leq 0$. But we already know from (9) that $0 \leq z(t) \leq 1$ for any $t \in[0, T]$. Thus, we must have $z(t) \in\{0,1\}$ for all $t \in[0, T]$.

If $\boldsymbol{x}(t) \in \operatorname{int}\left(\Omega_{1}\right)$ and $z(t)=0$, then it follows from (11) that $\omega(\boldsymbol{x}(t)) \leq 0$, which is a contradiction. Thus, if $\boldsymbol{x}(t) \in \operatorname{int}\left(\Omega_{1}\right)$, then $z(t)=1$. Similarly, if $\boldsymbol{x}(t) \in \operatorname{int}\left(\Omega_{2}\right)$, then $z(t)=0$. This shows that (9)-(11) implies (7). This completes the proof.

The technique described above for relaxing the binary-valued control is similar to the so-called embedding transformation in the literature $[16,23]$. Note, however, that we include the additional constraints (10) and (11) to ensure that the transformed system is equivalent to the original system. In the traditional embedding transformation, the two systems are not equivalent (only the bound constraints (9) are imposed in the traditional embedding transformation).

We can now rewrite the cost function (6) as follows:

$$
g_{0}(\boldsymbol{u}, z)=\sum_{k=1}^{m} \Phi_{0, k}\left(\boldsymbol{x}\left(\tau_{k} \mid \boldsymbol{u}, z\right)\right)+\int_{0}^{T} \mathcal{L}_{0}(\boldsymbol{x}(t \mid \boldsymbol{u}, z), \boldsymbol{u}(t)) d t .
$$

Similarly, the canonical constraints (4) and continuous inequality constraints (5) can be rewritten, respectively, as

$$
g_{v}(\boldsymbol{u}, z)=\sum_{k=1}^{m} \Phi_{v, k}\left(\boldsymbol{x}\left(\tau_{k} \mid \boldsymbol{u}, z\right)\right)+\int_{0}^{T} \mathcal{L}_{v}(\boldsymbol{x}(t \mid \boldsymbol{u}, z), \boldsymbol{u}(t)) d t \begin{cases}=0, & v=1, \ldots, p_{e}, \\ \geq 0, & v=p_{e}+1, \ldots, p,\end{cases}
$$

and

$$
h_{v}(\boldsymbol{x}(t \mid \boldsymbol{u}, z)) \geq 0, \quad t \in[0, T], \quad v=1, \ldots, q .
$$

On the basis of Proposition 3.1, it is clear that Problem 1 is equivalent to the following problem.

Problem 2 Choose a pair $(\boldsymbol{u}, z) \in \mathcal{U} \times \mathcal{Z}$ to minimize the cost function (12) subject to the dynamic system (8), the initial condition (2), the canonical constraints (13), and the continuous inequality constraints (10), (11), and (14). 


\section{Problem approximation}

Define a partition on the time horizon $[0, T]$ as follows:

$$
P=\left\{t_{0}, t_{1}, \ldots, t_{N}\right\}
$$

where $t_{0}=0, t_{N}=T$, and $t_{j-1} \leq t_{j}$ for each $j=1, \ldots, N$.

We assume that the partition $P$ is chosen so that for each $k \in\{1, \ldots, m\}$, there exists a corresponding $\nu_{k} \in\{1, \ldots, N\}$ such that $t_{\nu_{k}}=\tau_{k}$, i.e., the partition includes the characteristic times as node points.

We now approximate the control $\boldsymbol{u} \in \mathcal{U}$ by a piecewise constant function consistent with the partition $P$. In other words, the switching points for the piecewise constant control are the interior node points in the partition (i.e., $t_{j}, j=1, \ldots, N-1$ ). This piecewise constant approximation approach is an example of the well-known control parameterization technique in the literature [8].

The control switching points in the partition $P$ are decision variables to be chosen optimally. Although allowing the switching points to be variable increases the approximation accuracy, it unfortunately makes the problem more difficult to solve, as standard dynamic optimization methods, including those on which the optimal control software MISER 3.3 [5] is based, cannot deal directly with variable switching times [10]. Hence, we will apply the well-known time-scaling transformation described in $[3,6,20]$ to transform the variable switching times into fixed time points in a new time horizon. First, define

$$
\theta_{j}=t_{j}-t_{j-1}, \quad j=1, \ldots, N
$$

The time-scaling transformation is performed by defining a new time variable $s \in[0, N]$, and relating $s$ to $t$ through the following initial value problem,

$$
\begin{aligned}
& \dot{t}(s)=\theta_{j}, \quad s \in[j-1, j), \quad j=1, \ldots, N, \\
& t(0)=0,
\end{aligned}
$$

subject to the constraint

$$
t(N)=T \text {. }
$$

To ensure that the characteristic times are transformed correctly, we also require the following constraints in addition to (17):

$$
t\left(\nu_{k}\right)=\tau_{k}, \quad k=1, \ldots, m .
$$

For $s \in[j-1, j]$, integrating (15)-(16) yields

$$
t(s)=\sum_{l=1}^{j-1} \theta_{l}+\theta_{j}(s-j+1) .
$$

Let $\Theta$ be the set of all $\boldsymbol{\theta}=\left[\theta_{1}, \theta_{2}, \ldots, \theta_{N}\right]^{\top} \in \mathbb{R}^{N}$ satisfying $\theta_{j} \geq 0$ for each $j=1, \ldots, N$. 
Furthermore, let $\chi_{[j-1, j)}(s)$ be the indicator function defined by

$$
\chi_{[j-1, j)}(s)= \begin{cases}1, & \text { if } s \in[j-1, j) \\ 0, & \text { otherwise }\end{cases}
$$

Under the time-scaling transformation (15)-(16), the control switching times are mapped from $t=t_{1}, t_{2}, \ldots, t_{N-1}$ to $s=1,2, \ldots, N-1$. The approximate piecewise constant control can therefore be written as

$$
\tilde{\boldsymbol{u}}(s)=\sum_{j=1}^{N} \boldsymbol{\xi}_{j} \chi_{[j-1, j)}(s),
$$

where

$$
\boldsymbol{\xi}_{j} \in U, \quad j=1, \ldots, N
$$

We also assume that $z \in \mathcal{Z}$ is piecewise constant with respect to the partition $P$. Hence, in the new time horizon, we may write $z$ as

$$
\tilde{z}(s)=\sum_{j=1}^{N} \gamma_{j} \chi_{[j-1, j)}(s),
$$

where

$$
\gamma_{j} \in[0,1], \quad j=1, \ldots, N .
$$

Note that $N-1$ is an upper bound for the maximum number of mode switches.

We define

$$
\boldsymbol{\xi}=\left[\left(\boldsymbol{\xi}_{1}\right)^{\top},\left(\boldsymbol{\xi}_{2}\right)^{\top}, \ldots,\left(\boldsymbol{\xi}_{N}\right)^{\top}\right]^{\top}, \quad \boldsymbol{\gamma}=\left[\gamma_{1}, \gamma_{2}, \ldots, \gamma_{N}\right]^{\top}
$$

Let $\Xi$ be the set of all vectors $\boldsymbol{\xi}$ satisfying (19), and let $\Gamma$ be the set of all vectors $\gamma$ satisfying (20). In the new time horizon, the dynamic system (8) becomes

$$
\dot{\tilde{\boldsymbol{x}}}(s)=\gamma_{j} \theta_{j} \boldsymbol{f}^{1}\left(\tilde{\boldsymbol{x}}(s), \boldsymbol{\xi}_{j}\right)+\left(1-\gamma_{j}\right) \theta_{j} \boldsymbol{f}^{2}\left(\tilde{\boldsymbol{x}}(s), \boldsymbol{\xi}_{j}\right), \quad s \in[j-1, j), \quad j=1, \ldots, N,
$$

where $\tilde{\boldsymbol{x}}(s)=\boldsymbol{x}(t(s))$. Furthermore, the initial condition (2) becomes

$$
\tilde{\boldsymbol{x}}(0)=\boldsymbol{x}^{0} .
$$

Let $\tilde{\boldsymbol{x}}(\cdot \mid \boldsymbol{\xi}, \boldsymbol{\gamma}, \boldsymbol{\theta})$ be the solution of $(21)$ and (22) corresponding to $(\boldsymbol{\xi}, \boldsymbol{\gamma}, \boldsymbol{\theta}) \in \Xi \times \Gamma \times \Theta$. Therefore, our transformed cost function becomes

$$
\tilde{g}_{0}(\boldsymbol{\xi}, \boldsymbol{\gamma}, \boldsymbol{\theta})=\sum_{k=1}^{m} \Phi_{0, k}\left(\tilde{\boldsymbol{x}}\left(\nu_{k} \mid \boldsymbol{\xi}, \boldsymbol{\gamma}, \boldsymbol{\theta}\right)\right)+\sum_{j=1}^{N} \int_{j-1}^{j} \theta_{j} \mathcal{L}_{0}\left(\tilde{\boldsymbol{x}}(s \mid \boldsymbol{\xi}, \boldsymbol{\gamma}, \boldsymbol{\theta}), \boldsymbol{\xi}_{j}\right) d s
$$


Furthermore, the canonical constraints (13) are transformed to

$$
\begin{aligned}
\tilde{g}_{v}(\boldsymbol{\xi}, \boldsymbol{\gamma}, \boldsymbol{\theta})=\sum_{k=1}^{m} \Phi_{v, k}\left(\tilde{\boldsymbol{x}}\left(\nu_{k} \mid \boldsymbol{\xi}, \boldsymbol{\gamma}, \boldsymbol{\theta}\right)\right) \\
\quad+\sum_{j=1}^{N} \int_{j-1}^{j} \theta_{j} \mathcal{L}_{v}\left(\tilde{\boldsymbol{x}}(s \mid \boldsymbol{\xi}, \boldsymbol{\gamma}, \boldsymbol{\theta}), \boldsymbol{\xi}_{j}\right) d s \begin{cases}=0, & v=1, \ldots, p_{e}, \\
\geq 0, & v=p_{e}+1, \ldots, p .\end{cases}
\end{aligned}
$$

The continuous inequality constraints (14) become

$$
h_{v}(\tilde{\boldsymbol{x}}(s \mid \boldsymbol{\xi}, \boldsymbol{\gamma}, \boldsymbol{\theta})) \geq 0, \quad s \in[0, N], \quad v=1, \ldots, q .
$$

In addition, constraints (10) and (11) are transformed, respectively, to

$$
\tilde{G}_{j}\left(\gamma_{j}\right)=\gamma_{j}\left(\gamma_{j}-1\right) \geq 0, \quad j=1, \ldots, N,
$$

and

$$
\begin{array}{r}
\tilde{H}_{j}(s, \boldsymbol{\xi}, \boldsymbol{\gamma}, \boldsymbol{\theta})=\gamma_{j} \omega(\tilde{\boldsymbol{x}}(s \mid \boldsymbol{\xi}, \boldsymbol{\gamma}, \boldsymbol{\theta}))-\left(1-\gamma_{j}\right) \omega(\tilde{\boldsymbol{x}}(s \mid \boldsymbol{\xi}, \boldsymbol{\gamma}, \boldsymbol{\theta})) \geq 0, \\
s \in[j-1, j), \quad j=1, \ldots, N .
\end{array}
$$

We now define an approximate problem as follows.

Problem 3 Choose $(\boldsymbol{\xi}, \boldsymbol{\gamma}, \boldsymbol{\theta}) \in \Xi \times \Gamma \times \Theta$ to minimize the cost function (23) subject to the switched system (21), the initial condition (22), and the constraints (17), (18), and (24)-(27).

Note that, due to the piecewise constant assumption on the control, Problem 3 is only an approximation of Problem 2.

Standard numerical optimization algorithms will struggle with Problem 3 because constraint (26) defines a disconnected feasible region. In the next section, we introduce an exact penalty method to overcome this difficulty.

\section{Exact penalty method}

We apply the exact penalty approach in $[9,20-22]$ to transform Problem 3 into an unconstrained optimization problem that can be easily solved using the optimal control software MISER 3.3.

The constraint violation for Problem 3 is defined by

$$
\begin{aligned}
\Delta(\boldsymbol{\xi}, \boldsymbol{\gamma}, \boldsymbol{\theta}) & =\sum_{v=1}^{p_{e}} \tilde{g}_{v}(\boldsymbol{\xi}, \boldsymbol{\gamma}, \boldsymbol{\theta})^{2}+\sum_{v=p_{e}+1}^{p} \min \left\{\tilde{g}_{v}(\boldsymbol{\xi}, \boldsymbol{\gamma}, \boldsymbol{\theta}), 0\right\}^{2} \\
& +\sum_{v=1}^{q} \int_{0}^{N} \min \left\{h_{v}(\tilde{\boldsymbol{x}}(s \mid \boldsymbol{\xi}, \boldsymbol{\gamma}, \boldsymbol{\theta})), 0\right\}^{2} d s+\sum_{j=1}^{N} \min \left\{\tilde{G}_{j}\left(\gamma_{j}\right), 0\right\}^{2} \\
& +\sum_{j=1}^{N} \int_{j-1}^{j} \min \left\{\tilde{H}_{j}(s, \boldsymbol{\xi}, \boldsymbol{\gamma}, \boldsymbol{\theta}), 0\right\}^{2} d s+\sum_{k=1}^{m}\left[t\left(\nu_{k}\right)-\tau_{k}\right]^{2}+[t(N)-T]^{2} .
\end{aligned}
$$


Note that $\Delta(\boldsymbol{\xi}, \boldsymbol{\gamma}, \boldsymbol{\theta})=0$ if and only if constraints (17), (18) and (24)-(27) are satisfied.

Based on the constraint violation (28), using the strategy introduced in [9, 20-22], an exact penalty function is defined as follows:

$$
\hat{J}_{\delta}(\boldsymbol{\xi}, \boldsymbol{\gamma}, \boldsymbol{\theta}, \epsilon)= \begin{cases}\tilde{g}_{0}(\boldsymbol{\xi}, \boldsymbol{\gamma}, \boldsymbol{\theta}), & \text { if } \epsilon=0 \text { and } \Delta(\boldsymbol{\xi}, \boldsymbol{\gamma}, \boldsymbol{\theta})=0 \\ \tilde{g}_{0}(\boldsymbol{\xi}, \boldsymbol{\gamma}, \boldsymbol{\theta})+\epsilon^{-\alpha} \Delta(\boldsymbol{\xi}, \boldsymbol{\gamma}, \boldsymbol{\theta})+\delta \epsilon^{\eta}, & \text { if } \epsilon>0 \\ +\infty, & \text { otherwise }\end{cases}
$$

where $\epsilon$ is a new decision variable, $\delta>0$ is the penalty parameter and $\alpha$ and $\eta$ are positive constants satisfying $1 \leq \eta \leq \alpha$.

The new decision variable $\epsilon$ is subject to the following bound constraint:

$$
0 \leq \epsilon \leq \bar{\epsilon}
$$

where $\bar{\epsilon}>0$ is a small positive number.

We now define the following unconstrained problem.

Problem 4 Choose $(\boldsymbol{\xi}, \boldsymbol{\gamma}, \boldsymbol{\theta}) \in \Xi \times \Gamma \times \Theta$ and $\epsilon \in[0, \bar{\epsilon}]$ to minimize the penalty function (29) subject to the switched system (21) and the initial condition (22).

Note that when the penalty parameter $\delta$ is large, the third term $\delta \epsilon^{\eta}$ in $\hat{J}_{\delta}$ forces $\epsilon$ to be small, thus causing the second term $\epsilon^{-\alpha} \Delta(\boldsymbol{\xi}, \boldsymbol{\gamma}, \boldsymbol{\theta})$ to severely penalize constraint violations. This is why Problem 4 is a good penalty formulation for Problem 3.

To be more precise, for each $\delta$, let $\left(\boldsymbol{\xi}_{\delta}^{*}, \boldsymbol{\gamma}_{\delta}^{*}, \boldsymbol{\theta}_{\delta}^{*}, \epsilon_{\delta}^{*}\right)$ be a global solution of Problem 4 . Then according to Theorem 3 of [9], any sequence $\left\{\left(\boldsymbol{\xi}_{\delta}^{*}, \boldsymbol{\gamma}_{\delta}^{*}, \boldsymbol{\theta}_{\delta}^{*}\right)\right\}$ generated by an increasing sequence of penalty parameters contains a limit point as $\delta \rightarrow \infty$, and this limit point is a global solution of Problem 3.

In general, Problem 4 is non-convex and can only be solved locally. In this case, if $\left(\boldsymbol{\xi}_{\delta}^{*}, \boldsymbol{\gamma}_{\delta}^{*}, \boldsymbol{\theta}_{\delta}^{*}, \epsilon_{\delta}^{*}\right)$ is only a local solution of Problem 4 , then Theorem 4 of [9] guarantees that $\left(\boldsymbol{\xi}_{\delta}^{*}, \boldsymbol{\gamma}_{\delta}^{*}, \boldsymbol{\theta}_{\delta}^{*}\right)$ is a local solution of Problem 3 when $\delta$ is sufficiently large.

The convergence results stated above (which are proved in [9]) show that a solution of Problem 3 can be obtained by solving Problem 4 for large values of the penalty parameter. In the next section, we demonstrate the effectiveness of this method with a numerical example.

\section{Numerical example}

We consider the cancer chemotherapy problem in [11-13]. This problem involves choosing a chemotherapy administration regime to minimize the size of a cancerous tumour. Let $C(t)$ denote the number of cancer cells in the tumour at time $t$, and let $D(t)$ denote the concentration of anti-cancer drug at the tumour site at time $t$. Here, $t$ is measured in units of days. The drug concentration is modelled by the linear differential equation

$$
\begin{aligned}
\dot{D}(t) & =u(t)-\beta D(t), \\
D(0) & =0,
\end{aligned}
$$

where $u(t)$ is the drug delivery rate at time $t$ and $\beta$ is a given constant. According to this linear model, the drug concentration at the cancer site decreases exponentially after the drug is administered. 
The tumour growth rate is the net change due to cell proliferation (modelled using the Gompertz equation) and cell death from the anti-cancer drug (only active when the drug concentration is above a certain threshold):

$$
\begin{aligned}
& \dot{C}(t)=\underbrace{\lambda C(t) \ln (\mu / C(t))}_{\text {cell proliferation }}-\underbrace{ \begin{cases}0, & \text { if } D(t) \leq D_{\mathrm{th}}, \\
\kappa\left(D(t)-D_{\mathrm{th}}\right) C(t), & \text { if } D(t) \geq D_{\mathrm{th}}\end{cases} }_{\text {cell death due to anti-cancer drug }} \\
& C(0)=C_{0},
\end{aligned}
$$

where $C_{0}$ is the size of the initial tumour population, $D_{\text {th }}$ is the drug threshold concentration, and $\lambda, \mu$, and $\kappa$ are given constants. The cancer treatment commences at time $t=0$ and ends at time $t=T$. The objective is to minimize $C(T)$, the number of cancer cells at the terminal time.

The following constraints are imposed on the drug concentration to limit patient side effects:

$$
0 \leq D(t) \leq D_{\max }, \quad t \in[0, T],
$$

and

$$
\int_{0}^{T} D(t) d t \leq D_{\text {total }}
$$

where $D_{\max }$ and $D_{\text {total }}$ are given constants. As in [11-13], we also impose the following restrictions on the tumour size to ensure that it decreases at, or faster than, a specified rate:

$$
C\left(\tau_{k}\right) \leq \rho C\left(\tau_{k-1}\right), \quad k=1,2,3,
$$

where $\rho \in(0,1)$ is a given fraction and

$$
\tau_{k}=\frac{1}{4} k T, \quad k=0,1,2,3,4 .
$$

Since the magnitude of $C(t)$ is massive (in the order of $10^{10}$ ), we re-scale the problem by defining new state variables $x_{1}(t)=\ln (\mu / C(t))$ and $x_{2}(t)=D(t)$. The problem dynamics (30)-(33) then become

$$
\begin{aligned}
& \dot{x}_{1}(t)=-\lambda x_{1}(t)+ \begin{cases}0, & \text { if } x_{2}(t) \leq D_{\mathrm{th}}, \\
\kappa\left(x_{2}(t)-D_{\mathrm{th}}\right), & \text { if } x_{2}(t) \geq D_{\mathrm{th}},\end{cases} \\
& \dot{x}_{2}(t)=u(t)-\beta x_{2}(t),
\end{aligned}
$$

with initial conditions

$$
x_{1}(0)=\ln \left(\mu / C_{0}\right), \quad x_{2}(0)=0 .
$$

We also impose an upper bound on the drug delivery rate:

$$
0 \leq u(t) \leq u_{\max }, \quad t \in[0, T] .
$$


In terms of the new state variables, constraints (34)-(36) become

$$
\begin{aligned}
& 0 \leq x_{2}(t) \leq D_{\max }, \quad t \in[0, T] \\
& \int_{0}^{T} x_{2}(t) d t \leq D_{\text {total }}, \\
& x_{1}\left(\tau_{k}\right)-x_{1}\left(\tau_{k-1}\right)+\ln \rho \geq 0, \quad k=1,2,3 .
\end{aligned}
$$

The problem is to minimize $g_{0}=-x_{1}(T)$ (equivalent to minimizing the tumour size) subject to the dynamics given by (37) and (38), the initial conditions (39) and the constraints given by (40)-(43). In our simulations, we used the following values (taken from [11]) for the model constants:

$$
\begin{aligned}
& T=84.0, \quad \lambda=9.9 \times 10^{-4}, \quad \kappa=8.4 \times 10^{-3}, \quad \beta=0.27, \quad D_{\text {th }}=10.0, \\
& D_{\max }=50.0, \quad D_{\text {total }}=2.1 \times 10^{3}, \quad \rho=0.5, \quad \mu=10^{12}, \quad C_{0}=10^{10}, \quad u_{\max }=50.0 .
\end{aligned}
$$

After transforming and approximating the optimization problem using the procedures described in Sections 3-5 (with $N=32$ as the partition size), we obtained an unconstrained penalty problem. In this unconstrained problem, the goal is to minimize the penalty function (29), which includes the following terms to replace constraints (41), (42), and (43).

- Constraints (41):

$$
\begin{aligned}
\int_{0}^{T} \min \left\{x_{2}(t), 0\right\}^{2} d t & +\int_{0}^{T} \min \left\{D_{\max }-x_{2}(t), 0\right\}^{2} d t \\
& \longrightarrow \int_{0}^{N} \min \left\{\tilde{x}_{2}(s), 0\right\}^{2} d s+\int_{0}^{N} \min \left\{D_{\max }-\tilde{x}_{2}(s), 0\right\}^{2} d s
\end{aligned}
$$

- Constraint (42):

$$
\min \left\{D_{\text {total }}-\int_{0}^{T} x_{2}(t) d t, 0\right\}^{2} \longrightarrow \min \left\{D_{\text {total }}-\sum_{j=1}^{N} \int_{j-1}^{j} \theta_{j} \tilde{x}_{2}(s) d s, 0\right\}^{2} .
$$

- Constraints (43):

$$
\sum_{k=1}^{3} \min \left\{x_{1}\left(\tau_{k}\right)-x_{1}\left(\tau_{k-1}\right)+\ln \rho, 0\right\}^{2} \longrightarrow \sum_{k=1}^{3} \min \left\{\tilde{x}_{1}\left(\nu_{k}\right)-\tilde{x}_{1}\left(\nu_{k-1}\right)+\ln \rho, 0\right\}^{2} .
$$

The last term (corresponding to constraints (43)) is a nonlinear function involving the state value at two characteristic times. Since the optimal control software MISER 3.3 [5] requires that each nonlinear term involves only one characteristic time, we introduce two additional variables $\zeta_{1}$ and $\zeta_{2}$, where

$$
\zeta_{1}=\tilde{x}_{1}\left(\nu_{1}\right), \quad \zeta_{2}=\tilde{x}_{1}\left(\nu_{2}\right),
$$

where $s=\nu_{1}$ corresponds to $t=\tau_{1}$, and $s=\nu_{2}$ corresponds to $t=\tau_{2}$. Then the penalty 


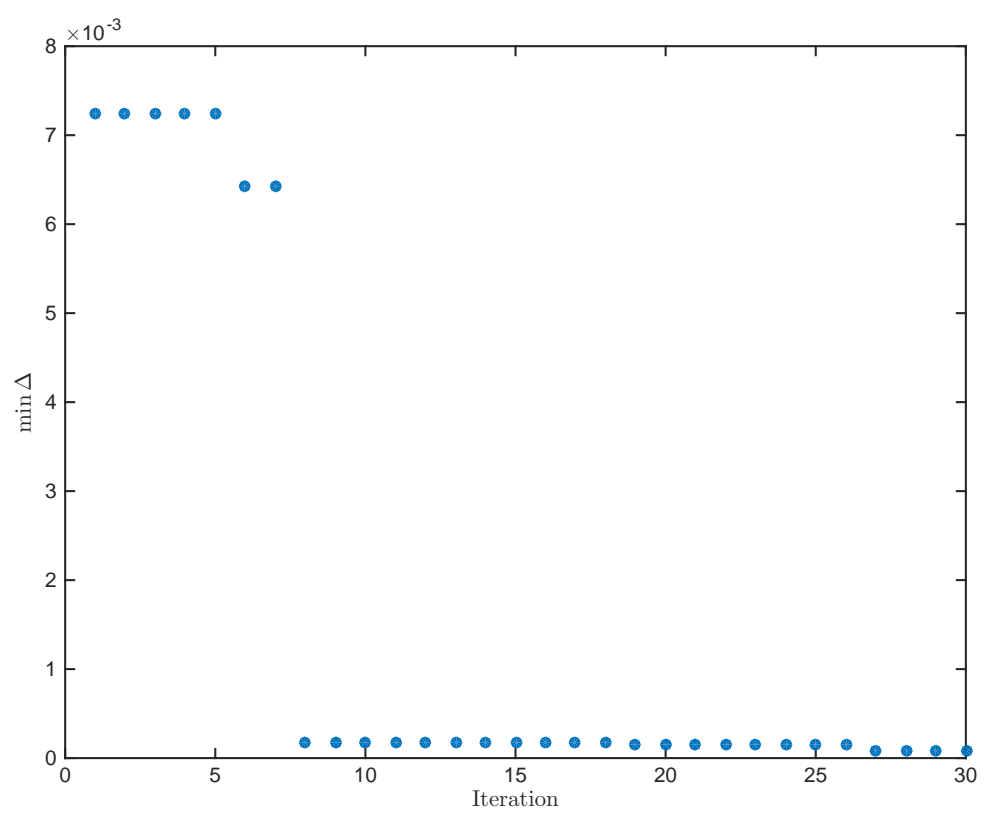

Figure 1. Numerical convergence of the exact penalty method starting from 30 random points: each dot shows the constraint violation of the best solution found (in terms of minimum constraint violation) up to the current iteration.

function term corresponding to (43) can be written as

$$
\begin{aligned}
\min \left\{\tilde{x}_{1}\left(\nu_{1}\right)-\ln \left(\mu / C_{0}\right)+\right. & \ln \rho, 0\}^{2}+\min \left\{\tilde{x}_{1}\left(\nu_{2}\right)-\zeta_{1}+\ln \rho, 0\right\}^{2} \\
& +\min \left\{\tilde{x}_{1}\left(\nu_{3}\right)-\zeta_{2}+\ln \rho, 0\right\}^{2}
\end{aligned}
$$

This format can be easily specified within MISER 3.3. The variables $\zeta_{1}$ and $\zeta_{2}$ are considered additional decision parameters subject to the canonical constraints (44). Note that these new canonical constraints also need to be added into the constraint violation (28) when solving the penalty problem using MISER 3.3. Any problem involving multiple characteristic times will require a similar transformation before MISER 3.3 can be applied, as the exact penalty method will result in a nonlinear function involving the state at each characteristic time; see the definition of the constraint violation (28).

We used MISER 3.3 to solve the penalty problem (with $\delta=1000, N=32, \alpha=3$, and $\eta=2$ ) starting from 30 randomly-chosen starting points. The numerical convergence of the constraint violation over these 30 iterations is shown in Figure 1. The details of the best solution obtained are reported in Table 1. Figures 2-4 show, respectively, the drug concentration, drug delivery rate, and tumour growth corresponding to the optimal solution. The graph in Figure 4 indicates that the cancer cell population is reduced significantly by the end of the treatment period.

Note that the results here are not directly comparable with [11-13] because these references do not consider the control upper bound (40). In the absence of this constraint, the optimal treatment regime involves short periods of high-intensity treatment around each characteristic time, leading to a graph of $u(t)$ with tall "spikes" near each characteristic time. We have included the upper bound constraint to make the results more realistic, at the expense of an increase in the final tumour size. 


\begin{tabular}{cccc}
\hline$x_{1}(T)$ & Final Tumour Population & $\epsilon^{*}$ & Constraint Violation \\
\hline 16.30357 & $8.3071 \times 10^{4}$ & 0.101811 & $7.2363 \times 10^{-3}$ \\
\hline
\end{tabular}

Table 1. Optimal solution for the cancer chemotherapy problem, where $\epsilon^{*}$ is the optimal value of $\epsilon$.

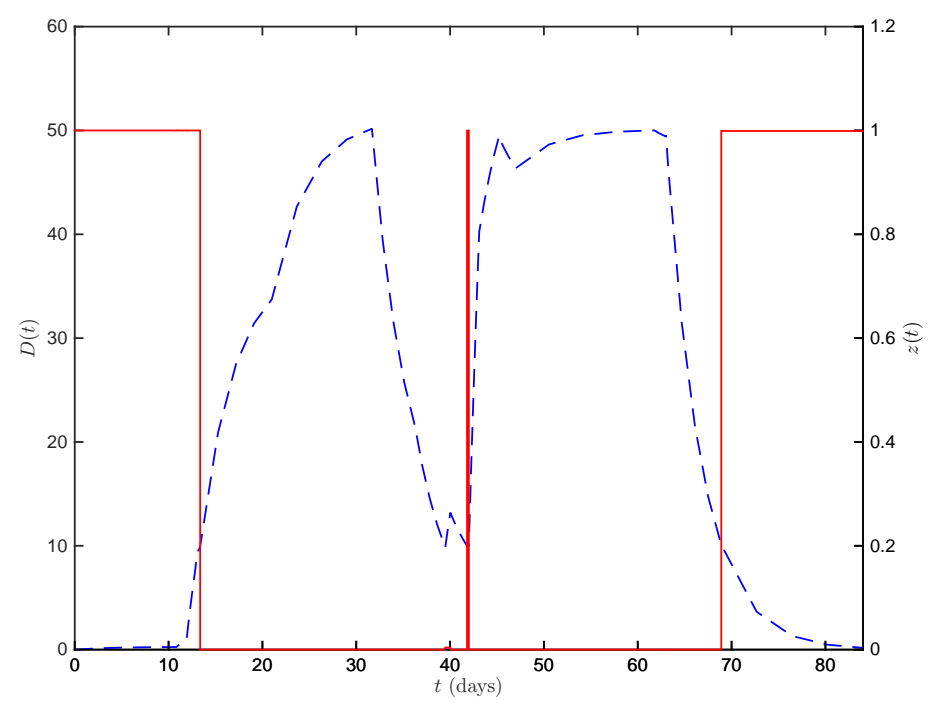

Figure 2. Drug concentration (blue dashed line) and optimal switching profile (red solid line) corresponding to the optimal solution of the cancer chemotherapy problem.

\section{Conclusion}

We have presented a new computational method for optimizing a class of dual-mode hybrid systems with state-dependent switching conditions subject to canonical and continuous inequality constraints. Our new method involves first introducing a binary-valued control function to express the hybrid system as a single system of ordinary differential equations. Then, we apply the control parameterization method (to approximate the continuous-time input signals) and the time-scaling transformation (to map the switching times to fixed points) to derive a finite-dimensional nonlinear dynamic optimization problem. This problem can be solved using the exact penalty method described in Section 5. Our numerical results for the cancer chemotherapy problem in [11-13] show that the proposed method is successful at solving realistic applied problems. Future work will involve extending the method in this paper to hybrid systems with more than two modes, and exploring more advanced multi-start strategies for dealing with non-convexity (for example, the particle swarm method used in [18] may be applicable).

\section{References}

[1] M. Boccadoro, Y. Wardi, M. Egerstedt, and E. Verriest, Optimal control of switching surfaces in hybrid dynamical systems, Discrete Event Dyn. Syst. 15 (2005), pp. 433-448.

[2] C.G. Cassandras, D.L. Pepyne, and Y. Wardi, Optimal control of a class of hybrid systems, IEEE Trans. Automat. Control 46 (2001), pp. 398-415. 


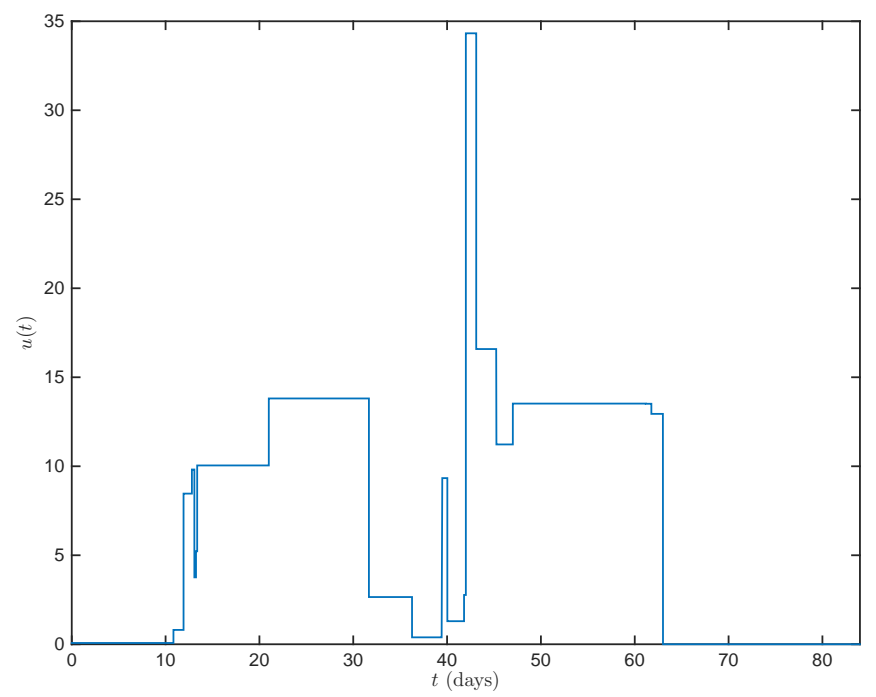

Figure 3. Optimal drug delivery rate for the cancer chemotherapy problem.

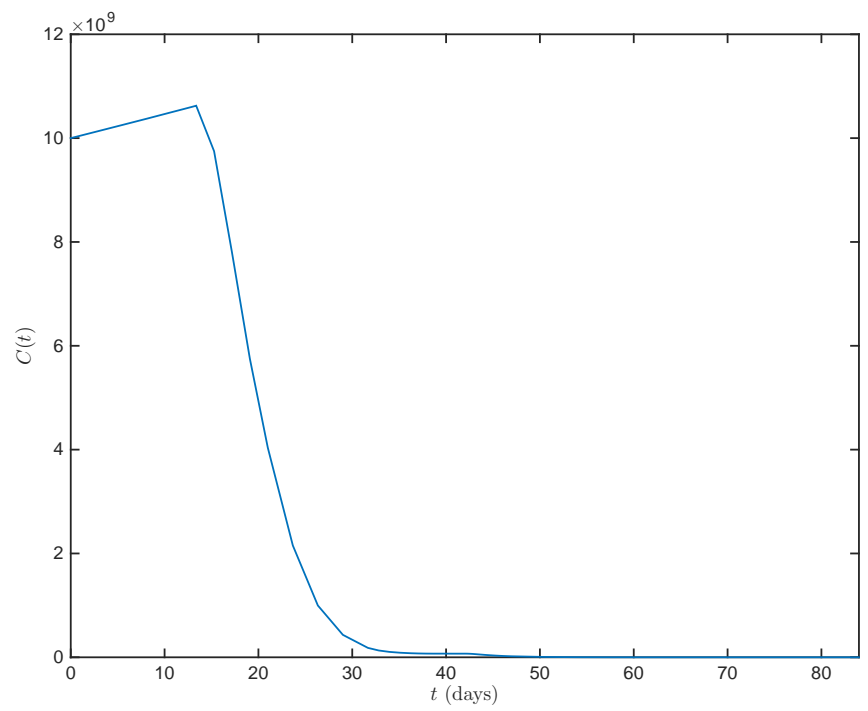

Figure 4. Number of tumour cells corresponding to the optimal drug delivery rate.

[3] Q. Chai, R. Loxton, K.L. Teo, and C. Yang, A max-min control problem arising in gradient elution chromatography, Ind. Eng. Chem. Res. 51 (2012), pp. 6137-6144.

[4] S. Hedlund and A. Rantzer, Optimal control of hybrid systems, in Proceedings of the 38th IEEE Conference on Decision and Control, Phoenix, USA, 7-10 December 1999, pp. 3972-3977.

[5] L.S. Jennings, M.E. Fisher, K.L. Teo, and C.J. Goh, MISER 3.3 Optimal Control Software - Theory and User Manual, University of Western Australia, Perth, Australia, 2004.

[6] H.W.J. Lee, K.L. Teo, V. Rehbock, and L.S. Jennings, Control parametrization enhancing technique for optimal discrete-valued control problems, Automatica J. IFAC 35 (1999), pp. 1401-1407.

[7] Q. Lin, R. Loxton, and K.L. Teo, Optimal control of nonlinear switched systems: Computational methods and applications, J. Oper. Res. Soc. China 1 (2013), pp. 275-311. 
[8] Q. Lin, R. Loxton, and K.L. Teo, The control parameterization method for nonlinear optimal control: A survey, J. Ind. Manag. Optim. 10 (2014), pp. 275-309.

[9] Q. Lin, R. Loxton, K.L. Teo, Y.H. Wu, and C. Yu, A new exact penalty method for semi-infinite programming problems, J. Comput. Appl. Math. 261 (2014), pp. 271-286.

[10] R. Loxton, Q. Lin, and K.L. Teo, Switching time optimization for nonlinear switched systems: Direct optimization and the time-scaling transformation, Pac. J. Optim. 10 (2014), pp. 537-560.

[11] R. Loxton, K.L. Teo, and V. Rehbock, Optimal control problems with multiple characteristic time points in the objective and constraints, Automatica J. IFAC 44 (2008), pp. 2923-2929.

[12] R.B. Martin, Optimal control drug scheduling of cancer chemotherapy, Automatica J. IFAC 28 (1992), pp. 1113-1123.

[13] R.B. Martin and K.L. Teo, Optimal Control of Drug Administration in Cancer Chemotherapy, World Scientific, Singapore, 1994.

[14] B. Passenberg, Theory and Algorithms for Indirect Methods in Optimal Control of Hybrid Systems, Ph.D. Thesis, Technical University of Munich, Munich, Germany, 2012.

[15] T. Ruby, V. Rehbock, and W.B. Lawrance, Optimal control of hybrid power systems, Dyn. Contin. Discrete Impuls. Syst. Ser. B Appl. Algorithms 10 (2003), pp. 429-439.

[16] D.K. Shin, Z. Gürdal, and O.H. Griffin, A penalty approach for nonlinear optimization with discrete design variables, Eng. Optim. 16 (1990), pp. 29-42.

[17] O. Stursberg and S. Panek, Control of switched hybrid systems based on disjunctive formulations, in Hybrid Systems: Computation and Control, C.J. Tomlin and M.R. Greenstreet, eds., Springer, Berlin, Germany, 2002, pp. 421-435.

[18] L. Wang, Q. Lin, R. Loxton, K.L. Teo, and G. Cheng, Optimal 1,3-propanediol production: Exploring the trade-off between process yield and feeding rate variation, J. Process Control 32 (2015), pp. 1-9.

[19] S.F. Woon, V. Rehbock, and R. Loxton, Towards global solutions of optimal discrete-valued control problems, Optimal Control Appl. Methods 33 (2012), pp. 576-594.

[20] C. Yu, B. Li, R. Loxton, and K.L. Teo, Optimal discrete-valued control computation, J. Global Optim. 56 (2013), pp. 503-518.

[21] C. Yu, K.L. Teo, and Y. Bai, An exact penalty function method for nonlinear mixed discrete programming problems, Optim. Lett. 7 (2013), pp. 23-38.

[22] C. Yu, K.L. Teo, L. Zhang, and Y. Bai, On a refinement of the convergence analysis for the new exact penalty function method for continuous inequality constrained optimization problem, J. Ind. Manag. Optim. 8 (2012), pp. 485-491.

[23] F. Zhu and P.J. Antsaklis, Optimal control of hybrid switched systems: A brief survey, Discrete Event Dyn. Syst. 25 (2015), pp. 345-364. 